\title{
Artrópodos: plaga presente en unidades de producción de Anturio para flor de corte en la zona centro de Veracruz, México
}

\author{
Emmanuel Herrera Martínez $z^{1}$ - Gabriel Otero Colina ${ }^{2}$ - \\ Rosaura Jose Pablo ${ }^{1}$ Juan A. Villanueva Jiménez ${ }^{1}$ \\ Colegio de Posgraduados en Ciencias Agrícolas, Campus Veracruz, México
}

Recepción: 29/06/016

Aceptación: 13/07/2016

\section{Resumen}

Existe escasa información de la artropofauna de importancia agrícola en Anturio. Por ello, se realizó la colecta e identificación de artrópodos que tienen el potencial como plagas y enemigos naturales, presentes en sistemas de producción de anturio de corte, en la zona Centro del Estado de Veracruz, México. El mayor número de organismos colectados es de la clase Acarida (913) que representa $60.5 \%$ del total; 589 individuos corresponden al orden Thysanoptera, (39.3\%), y siete a Hemiptera (Aphididae, 0.5\%). Además, se identificaron siete familias de ácaros: Tarsonemidae, Tetranychidae, Tenuipalpidae, Phytoseiidae, Stigmaeidae, Glyeyphagidae y Tydeidae. Esta información representa la base para el diseño de medidas integrales de manejo del agroecosistema.

Palabras clave: ácaros depredadores, ácaros plaga, insectos fitófago, insectos depredadores, trips.

\begin{abstract}
There is little information on arthropod fauna of agricultural importance in Anturio. For this reason, the collection and identification of arthropods that have the potential as pests and natural enemies, present in anthurium cutting systems, were carried out in the central area of the State of Veracruz, Mexico. The largest number of collected organs is about the mite kind (913) that represents $60.5 \%$ of the total; 589 individuals correspond to the Thysanoptera order, (39.3\%), and seven to Hemiptera (Aphididae, 0.5\%). In addition, seven families of mites were identified: Tarsonemidae, Tetranychidae, Tenuipalpidae, Phytoseiidae, Stigmaeidae, Glyeyphagidae and Tydeidae. This information represents the basis for the design of integrated agroecosystem management measures.
\end{abstract}

Key words: predatory mites, plague mites, phytophagous insects, predatory insects, thrips.

1. Colegio de Postgraduados, Campus Veracruz. Km 88.5 Carretera Xalapa-Veracruz, Manlio F. Altamirano, Veracruz, México. CP 91970.

2. Colegio de Postgraduados, Campus Montecillo. Km. 36.5 Carretera México-Texcoco, Montecillo, Texcoco, México. CP 56230. 


\section{Introducción}

En los agroecosistemas complejos con altos niveles de biodiversidad, por lo general existe mayor regulación de plagas, lo cual es propiciado por la gran variedad y abundancia de depredadores y parasitoides (Altieri, 1994). Por tanto se deben identificar las relaciones que existen entre las especies que, a través de sus sinergias, abastecen y promueven servicios ecológicos claves como el control biológico de plagas. Para aprovechar estas sinergias se deben diseñar y manejar agroecosistemas, con un entendimiento básico de las interacciones entre el suelo, las plantas, los artrópodos y los microorganismos (Altieri y Nicholls, 2007).

Las plantas ornamentales representan una opción productiva para los habitantes de la zona Centro de Veracruz, México. Los Anturios son flores tropicales adaptadas a este ambiente, con el género Anthurium como uno de los más sobresalientes. Pertenecen a la familia Araceae, que comprende más de 1500 especies tropicales (Farsi et al., 2012). Las especies de mayor interés son Anthurium andreanum Linden y A. scherzerianum Schott (Gantait y Mandal, 2010); sin embargo A. andreanum es la especie más importante desde el punto de vista económico, debido a que se vende como flor de corte, tiene la espata de diversos colores y presenta una larga duración después de que ha sido cortada; caracterís- ticas que le permite tener una alta demanda en el mercado nacional e internacional (López-Puc et al., 2013).

Los principales artrópodos fitófagos con reportes de daño al cultivo de anturio son: trips de las especies Chaetanaphothrips orchidii Moulton, C. signipennis (Bagnall) y Frankliniella occidentalis Pergande, las cuales afectan a la flor al producir rayas blancas y distorsiones de la espata (Hara et al., 2002; Bushe et al., 2004); la mosca blanca Aleurotulus anthuricola Nakahara, Trialeurodes vaporariorum Westwood y Bemisia tabaci Gennadius, estas son identificadas por secreciones blancas, polvo ceroso, que en infestaciones altas, las pupas se pueden presentar incluso en la espata; diversas especies del orden Orthoptera de la familia Acrididae, que ocasionan lesiones en las hojas al alimentarse (Anteparra et al., 2013). Además se han observado diferentes especies de pulgones (Hemiptera; Aphididae) en el envés de la hoja y botones florales, tales como Aphis gossypii Glover y Myzus persicae Sulzer; otras especies del orden son Hemiberlesia lataniae Signoret (Diaspidadae) y Planococcus citri Risso (Pseudococcidae) presentes en el envés de la hoja, generalmente asociadas con hormigas (Pato et al., 2007; Anteparra et al., 2013).

Entre los ácaros (clase Acarida) se citan a Brevipalpus phoenicis Geijskes (Tenuipalpidae), Tetranychus urticae Kock (Tetranyquidae) y 
Polyphagotarsonemus latus Banks (Tarsonemidae, quienes producen el bronceado del pecíolo y la espata (Ochoa et al., 1991; Dreistadt, 2001; Garita et al., 2014). Dada la necesidad de contar con información para el manejo del cultivo de anturio como actividad económica, se colectaron e identificaron las especies de artrópodos de importancia agrícola en sistemas de producción de Anturio de corte en la zona Central del Estado de Veracruz, México.

\section{Materiales y métodos}

Los sitios en donde se realizaron las colectas, se seleccionaron con base en el nivel de producción, altura sobre el nivel del mar y mediante entrevistas con los productores. Se integraron tres zonas, la primera con tres sitios a menos de 800 metros sobre el nivel del mar $(\mathrm{msnm})^{3}$ en el municipio de Tepatlaxco, Veracruz, México. La segunda concentra dos sitios en el municipio de Fortín de las Flores y uno en el municipio de Emiliano Zapata, Veracruz, que se encuentran entre 800 y 1000 msnm. La tercera zona incluye sitios a más de 1000msnm en los municipios de Sochiapa, Fortín de las Flores y Emiliano Zapata. La colecta se llevó a cabo del mes de junio a octubre de 2007, lo que permitió el registro de especies para la época de lluvias y la de estiaje. Las colectas se dirigieron a los botones florales, hojas jóvenes y adultas, con evidencias de daño por plaga.

En el caso de las colectas dirigidas a botones de Anturio, se cortaron tres botones florales por sitio en cada visita mensual. Los botones se conservaron en frascos de plástico cerrados con capacidad de 1L. La colecta en hojas se realizó mediante el método de lavado y tamizado; se utilizó un aspersor manual con capacidad de 2L y con tamices de 20, 40 y 400 mallas por pulgada. Los organismos colectados se depositaron en frascos de plástico de $50 \mathrm{~mL}$ con alcohol etílico al 70\% para el traslado al laboratorio.

Para la identificación de los ácaros se utilizó líquido de Hoyer como medio de montaje. Posteriormente se identificaron a través del microscopio de contraste de fases (Carl Zeiss Mod. Primo Star) y clave dicotómica para la identificación de los órdenes y familias de ácaros de importancia agrícola. La identificación a especie se realizó por el segundo autor, especialista en taxonomía de Acaridae, Dr. Gabriel Otero Colina, Profesor investigador del Colegio de Postgraduados, Campus Montecillo. La identificación de trips fue realizada por la Dra. Aurea Mojica Guzmán del Laboratorio de Entomología, departamento de Zoología del Instituto de Biología de la Universidad Autónoma de México. También con los productores asociados al Consejo Veracruzano de la Flor.

Finalmente, la información se sumariza en cuadros, por grupo de artrópodos de los tres sitios muestreados, así como figuras que presentan el porcentaje de organismos de artró- 
podo en los meses de junio (fin de época de estiaje), julio, agosto, septiembre y octubre (época de lluvia) de 2007.

\section{Resultados y discusión}

En general, el mayor porcentaje de organismos colectados en los botones de flor de Anturio de junio a octubre corresponde a la clase
Acarida. El segundo lugar en abundancia corresponde al orden Thysanoptera, con el mayor número de insectos presentes en el mes de junio. El orden Hemiptera (Aphididae) presentó el menor porcentaje de organismos, con su pico máximo en el mes de junio. En total se colectaron 1509 individuos de 135 botones florales de anturio (Figura 1).

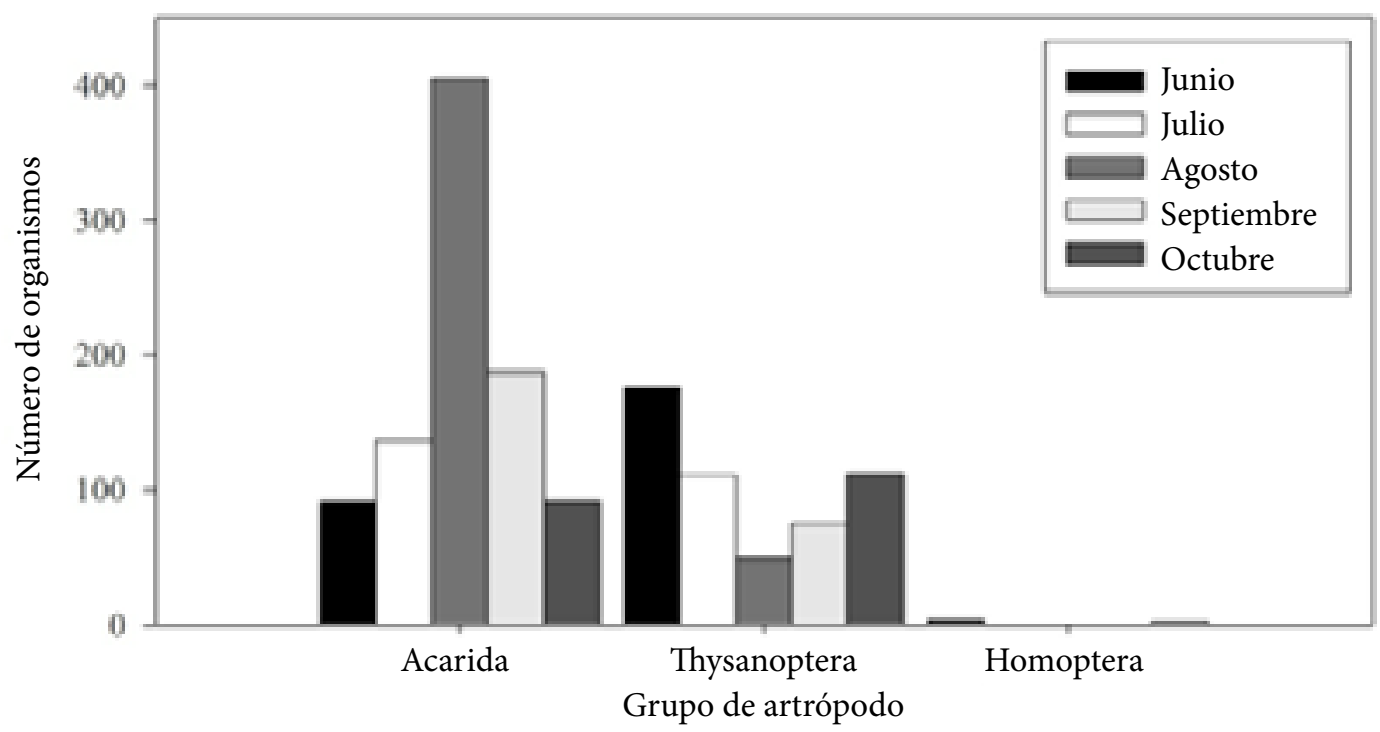

Figura 1. Porcentaje de organismos colectados en botones de flor de Anturio en tres sitios de muestreo de la zona Centro del Estado de Veracruz, México.

Se colectaron hasta 245 individuos de la clase Acarida en un solo botón. En promedio se encontraron $6.8 \pm 24.0$ (desviación estándar) individuos por botón. Dentro del orden Thysanoptera, la mayor cantidad de individuos corresponde al estado de larva, seguido por el de adulto. El número medio de larva presente por flor de anturio fue $5.2 \pm 12.0$; mientras que en estado adulto se encontró $1.1 \pm 2.0$ individuos. Con respecto a la familia Aphididae, se encontró un máximo de cuatro individuos en estado de ninfa, con un promedio de $0.1 \pm 0.0$ (Tabla 1 ). 
Tabla 1. Grupo de artrópodos colectados en 135 flores de Anturio con base en su estado de desarrollo, en tres sitios de muestreo en la zona Central Costera de Veracruz, México

\begin{tabular}{|l|c|c|c|c|}
\hline $\begin{array}{c}\text { Grupo de artrópodo/ } \\
\text { estado desarrollo }\end{array}$ & Media & $\begin{array}{c}\text { Desviación } \\
\text { estándar }\end{array}$ & $\begin{array}{c}\text { Mínimo/ } \\
\text { individuos }\end{array}$ & $\begin{array}{c}\text { Máximo/ } \\
\text { individuos }\end{array}$ \\
\hline Acarida & 6.8 & 24.0 & 0 & 245 \\
\hline Ácaro & \multicolumn{5}{|l}{} \\
\hline Thysanoptera & 5.2 & 19.0 & 0 & 133 \\
\hline Larva & 0.0 & 0.0 & 0 & 3 \\
\hline Prepupa & 0.1 & 1.0 & 0 & 12 \\
\hline Pupa & 1.1 & 2.0 & 0 & 19 \\
\hline Adulto & & & & 4 \\
\hline Hemiptera: Aphididae & 0.1 & 0.0 & 0 & 0 \\
\hline Ninfas & 0.0 & 0.0 & 0 & \\
\hline Adulto &
\end{tabular}

Los sitios que presentaron mayor porcentaje (50.03\%) de organismos del total de la colecta, fueron los municipios de Sochiapa, Fortín de las Flores y Emiliano Zapata que se sitúan a una altitud mayor a $1000 \mathrm{msnm}$; donde el mayor porcentaje de organismos en botones de flor de Anturio fue del orden Thysanoptera con $58.0 \%$, seguido de la clase Acarida y de la familia Aphididae con 41.1 y $0.9 \%$ individuos respectivamente (Figura 2); mientras que los sitios de los municipios de Fortín de la Flores y Emiliano Zapata que se encuentran entre los 800 y $1000 \mathrm{msnm}$, contuvieron el $28.23 \%$ del total de los organismos colectados.

En estos sitios, la clase Acarida fue la predominante en el botón de la flor de Anturio con $85.2 \%$ individuos, seguida del orden Thysanoptera con $14.8 \%$ organismos (Figura 2). Por último, en el sitio con altitud de 800msnm que corresponde al municipio de Tepatlaxco, se encontró el $21.74 \%$ de organismos colectados. Asimismo, la presencia de la clase Acarida en botón de la flor de Anturio fue mayor (73.2\%), comparado con el orden Thysanoptera (26.8\%) (Figura 2).

La presencia de los grupos de artrópodos de la clase Acárida, orden Thysanoptera y Hemiptera en cultivos de Anturio de las zonas muestreadas en este estudio; se confirma con los encontrados por San Juan (2010) en el mismo cultivo en la región Córdoba-Fortín de la Flores, donde se identificaron diez plagas principales en la producción de Anturio, entre las que citan a las arañas rojas, los trips y los pulgones. 


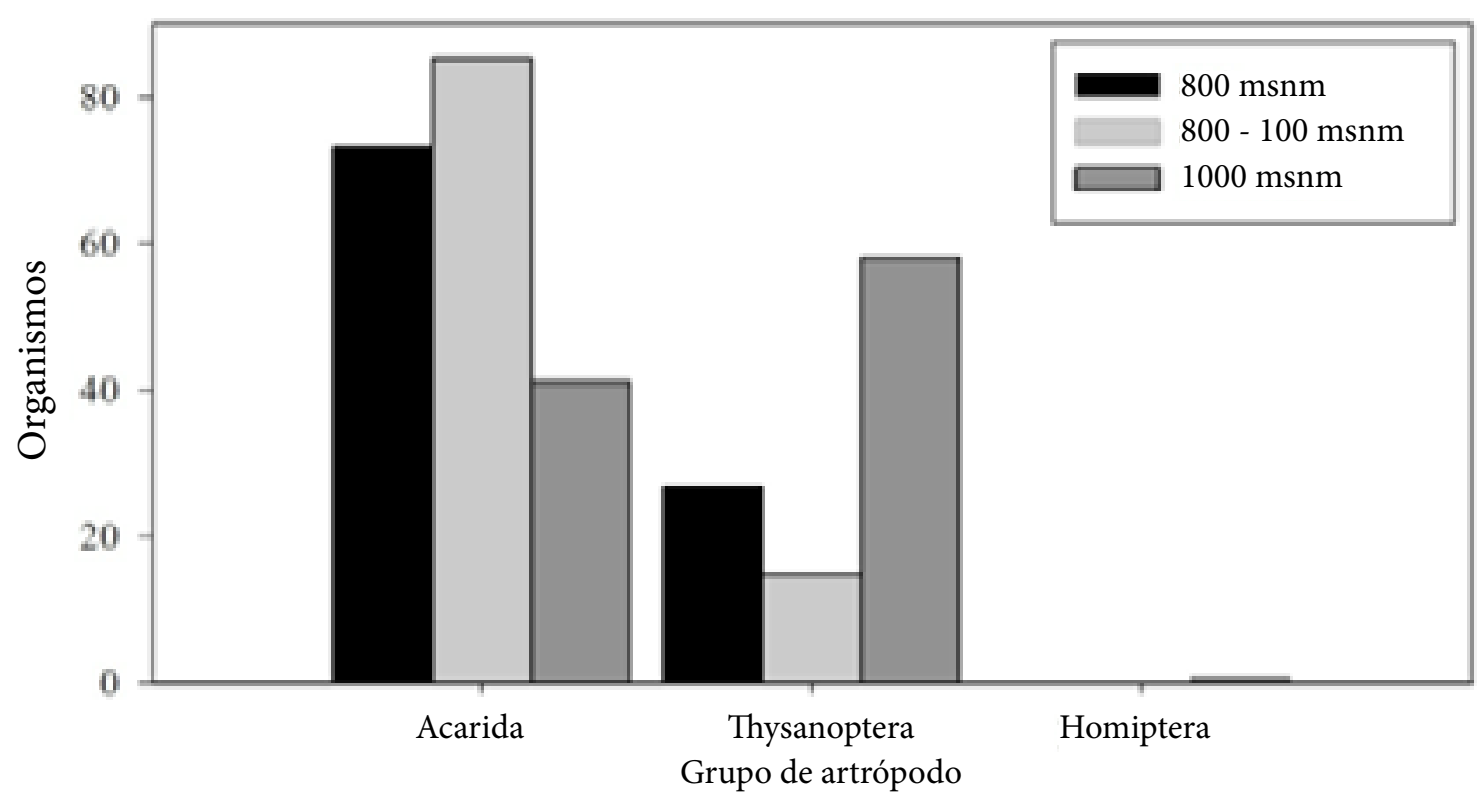

Figura 2. Porcentaje de organismos de artrópodos en botones de flor de Anturio, en tres zonas de muestreo con diferentes altitudes, Centro del Estado de Veracruz, México.

Resultados similares encontraron Bushe et al. (2004) y Anteparra et al. (2013), quienes identificaron diferentes artrópodos fitófagos asociados en diferentes órganos de la planta de Anturio, entre ellas del orden Hemiptera (Hemiptera), que fue observado en el envés de la hoja, botones florales y flores; además de insectos del orden Thysanoptera sobre flores jóvenes, con sus principales síntomas como rayas blancas y una distorsión que aparecen en la parte superior de la superficie inferior de la espata.

Dentro de la clase Acarida se identificaron siete familias, correspondientes a organismos fitófagos de las familias Tarsonemidae, Tetranychidae y Tenuipalpidae, las cuales agru- pan especies asociadas a cultivos agrícolas de importancia económica, así como a diversas especies de plantas ornamentales (Aguilar y Murillo, 2012; Fathipour y Maleknia, 2016). Algunas especies de ácaros de las familias Tetranychidae y Tenuipalpidae destacan por ser vectoras de enfermedades virales en diferentes cultivos, entre ellas plantas ornamentales que pertenecen al género Anthurium (Childers et al., 2003; Beltrán-Castillo et al., 2010; Kitajima et al., 2010; Mesa y Valencia, 2013).

Dentro la familia Tenuipalpidae se identificaron a las especies Brevipalpus abovatus Donnadieu y B. viquierae Baker. La primera se ha presenciado en una amplia gama de huéspedes; se alimenta de diferentes órganos de la 
planta (hojas, ramas, flores y frutos), además, es vectora de rhabdovirus en cultivos de cítricos, maracuyá, café, orquídeas y plantas ornamentales (Kitajima et al., 2003; Childers y Rodrigues, 2011). Evans et al. (1998) encontraron esporas de hongos pegadas a B. viquierae, por tal razón, esta especie podría tener un rol importante en la diseminación y desarrollo de hongos fitoparasíticos en cultivos.

Los organismos identificados como depredadores son de las familias Phytoseiidae y Stigmaeidae. Glycyphagidae y Tydeidae; familias que agrupan especies fungívoras, depredadoras y fitófagas. Los ácaros de las familias Phytoseiidae y Stigmaeidae presentes en Anturio son considerados como depredadores de ácaros plaga. Dentro de la familia Phytoseiidae, la mayoría de las especies depredan ácaros plaga, principalmente de la familia Tetranychidae, y en menor grado consumen especies de las familias Tarsonemidae y Tenuipalpidae en diversos cultivos (Badii et al., 2010; De la Torre-Santana, 2015).

Amblyseius cucumeris es una especie de fitoseidos que se ha utilizado como agente de control biológico en adultos de trips de Frankliniella occidentalis en Anturio (Rodríguez, 2007). En el cultivo de Anturio también se identificó la familia Tydeidae, ésta contiene especies plaga y benéficas en cultivos agrícolas; sin embargo, su importancia económica es menor en comparación con los tetraníqui- dos, tarsonémidos y eriófidos; como controladores biológicos también son menos efectivos que los ácaros fitoseidos y estigmaideos en el control de plagas. Las especies de esta familia son depredadores de ácaros plaga, principalmente del orden Mesostigmata, pero también son fungívoras, micófagas y carroñeras (Niemczyk y Kaźmierski, 2002; Akbari et al., 2015).

Las especies de trips presentes en Anturio, incluyeron a Chaetanaphothrips orchidii (Moulton), reportada previamente en Hawaii (Hara et al., 2002; Bushe et al., 2004), y Apterygothrips longiceps (Hood), aparentemente realizando actividad depredadora. Además, se localizaron dos nuevas especies, Tryphactothrips sp. nov., género que ha sido reportada en India, Taiwán y el Caribe, así como Pseudodendrothrips sp. nov., género que ha sido reportado en Europa, Asia, África, Australia y Norteamérica. Debido a lo poco estudiado del grupo, se requiere más investigación para determinar el alcance de estas especies nuevas y su actividad en Anturio. Además, la falta de adultos de los pulgones colectados, impidieron una correcta identificación a nivel de especie.

Esta es una primera aproximación a la identidad de los artrópodos plaga de los Anturios en la región centro de Veracruz, México. Hace falta continuar con la identificación de las especies presentes, determinar la biología 
de las mismas, revisar el papel de los controladores biológicos y verificar la extensión del daño de los fitófagos. A partir de ahí, se pueden incorporar métodos de control que permitan el manejo agroecológico del cultivo, o generar paquetes tecnológicos sustentables, adecuados al sistema de producción.

\section{Conclusiones}

Se identificaron tres grupos de artrópodos presentes en botones de flor de Anturio. Cuatro especies pertenecen al orden Thysanoptera, al menos una de ella depredadora. Al menos una especie fitófaga de la Familia Aphididae, orden Hemiptera. Del orden Acarida se reconocieron tres familias que comprenden ácaros fitófagos (Tarsonemidae, Tetranychidae y Tenuipalpidae) y dos familias que agrupan especies depredadores (Phytoseiidae y Stigmaeidae). Se requiere continuar con el trabajo para identificar todas las especies de artrópodos presentes, determinar su papel biológico y generar un paquete tecnológico de manejo sustentable de las plagas de Anturio.

\section{Referencias}

Aguilar, H. y Murillo, P. (2012). Nuevos hospederos y registros de ácaros fitófagos para Costa Rica: Período 2008-2012. Agronomía Costarricense, Vol. 36, p. 11-28. Recuperado de http:// www.mag.go.cr/rev_agr/index.html

Akbari, A., Irani-Nejad, K. H., Khanjani, M., Arzanlou, M. y Kaźmierski, A. (2015). A new tydeid species (Acari: Tydeidae) with a key to Brachtydeus species from East Azerbaijan Province, Iran. Systematic \& Applied Acarology, vol. 20, p. 423-430. Doi: http://dx.doi. org/10.11158/saa.20.4.7

Altieri, M. A. (1994). Biodiversity and Pest Management in Agroecosystems. Haworth Press, N. Y. 185.

Altieri, M. A. y Nicholls, C. I. (2007). Conversión agroecológica de sistemas convencionales de producción: teoría, estrategia y evaluación. Ecosistemas, Vol. 16, p.3-12. Recuperado de http:// www.revistaecosistemas.net/artículo.asp? $\mathrm{Id}=457$

Anteparra, M., Loayza, A., Granados, L., y Díaz, W. (2013). Insectos asociados con anturio (Anthurium andreanum Lind.) en Tingo María, Perú. Investigación y Amazonía, Vol. 3, p. 8493. Recuperado de: file://C:/Users/usuario/Downloads/51-203-1-PB\%20(2).pdf 
Badii, M. H., Landeros, J. y Cerna E. (2010). Regulación poblacional de ácaros plaga de impacto agrícola. Daena: Internacional Journal of Good Conscience, Vol. 5, p.270-302. Recuperado de: http://www.spentamexico.org/v5-n1/5(1)270-302.pdf

Beltrán-Castillo, A., Díaz, Y., Otero-Pujo, L., Borges-Soto, M., Pérez-Artiles, L., Avalos-Rodríguez, Y., González-Arias, G., Hernández-Espinosa, D., Rodríguez Tapia, J. L., y De la TorreSantana, P. (2010). Importancia de los ácaros del género Brevipalpus (Acari: Tenuipalpidae) y las plantas hospedantes asociadas. Revista CitriFrut, vol. 27, p. 54-59. Recuperado de: http:// agris.fao.org/agris-search/search.do?recordID=CU2011900008

Bushe, B. C., Nishijima, W. T., Hara, A. H., y Sato, D. M. (2004). Identifying anthurium flower injuries. Plant Disease. CTAHR. University of Hawai'i at Mânoa. P.; p: 1-7. Recuperado de: http://hdl.handle.net/10125/12401

Childers, C. C. y Rodrigues, J. C. V. (2011). An overview of Brevipalpus mites (Acari: Tenuipalpidae) and the plant viruses they transmit. Zoosymposia, Vol. 6, p. 180-192. Recuperado de http:// www.mapress.com/zoosymposia/

Childers, C. C., French, J. V., y Rodrigues, J. C. (2003). Brevipalpus californicus, B. obovatus, B. phoenicis, and B. lewisi (Acari: Tenuipalpidae): a review of their biology, feeding injury and economic importance. Experimetal and Applied Acarology, vol. 30, p. 5-28. Recuperado de https://www.ncbi.nlm.nih.gov/pubmed/14756411

De la Torre-Santana, P. E. (2015). La familia Stigmaeidae (Acari: Trombidiformes) en Cuba. Revista de Protección Vegetal, vol. 30. Recuperado de: http://scielo.sld.cu/scielo.php?script=sci arttext\&pid=S1010-27522015000400045

Dreistadt, S. H. (2001). Integrated pest management for floriculture and nurseries. University of California; p. 423

Evans, G. A., Cromroy, H. L., y Ochoa, R. (1998). The family Tenuipalpidae in Bermuda (Prostigmata: Acari). Florida Entomologist, Vol. 81, p. 167-170. Recuperado de http://www. jstor.org/stable/3496082?seq=1\#page_scan_tab_contents

Farsi, M.; Taghavizadeh, Y. M. E., y Qasemiomran, V. (2012). Micropagation of Anthurium adreanum cv. Terra. African Journal of Biotechnology, Vol. 11, p. 13162-13166. Recuperado de Doi: 10.5897/AJB12.893 
Fathipour, Y. y Maleknia, B. (2016). Mite Predators; p. 329-366. Recuperado de: http://dx.doi. org/10.1016/B978-0-12-803265-7.00011-7

Gantait, S. y Mandal, N. (2010). An elite protocol for accelerated quality-cloning in Gerbera jamesonii Bolus cv. Sciella. In Vitro Cellular \& Develomental Biology-Plant, Vol. 46, p. 537-548. doi: 10.1007/s11627-010-9319-2

Garita, L. C., Tassi, A. D., Calegario, R. F., Freitas-Astúa, J., Salaroli, R. B., Româo, G. O. y Kitajima, E. W. (2014). Experimental host range of Citrus leprosis virus C (CiLV-C). Tropical Plant Pathology, vol. 39, p. 043-055. Recuperado de: http://www.scielo.br/scielo.php?script=sci_artte xt\&pid=S1982-56762014000100006

Hara, A. H., Jacobsen, C. y Niino-DuPonte, R. (2002). Anthurium trips damage to ornamentals in Hawaii. Insect Pests. CTAHR. Department of Agriculture. University of Hawai'I at Mânoa. Recuperado de http://www.ctahr.hawaii.edu/oc/freepubs/pdf/ip-9.pdf

Kitajima, E. W. Chagas, C. M. y Rodrigues, J. C. V. (2003). Brevipalpus-transmitted plant viruslike diseases: cytopathology and some recent cases. Experimental and Applied Acarology; vol. 30, p. 135-160. Doi: 10.1023/B:APPA.0000006546.55305.e3

Kitajima, E. W., Rodrigues, C. V. y Freitas-Astua, J. (2010.) An annotated list of ornamentals naturally found infected by Brevipalpus mite-transmitted viruses. Scientia Agricola, Vol. 67, p. 348-371. Doi: http://dx.doi.org/10.1590/S0103-90162010000300014

López-Puc, G.; Ramírez-Mosqueda, M. A. y Lee, E. H. E. (2013). El cultivo moderno del anturio. En Ciencia, vol. 64, p. 52-59. Recuperado de http://www.revistaciencia.amc.edu.mx/images/revista/64_3/PDF/Anturio.pdf

Mesa, N. C., y Valencia, M. O. (2013). Diagnóstico taxonómico de la familia Tenuipalpidae (Acari: Tetranychoidea) en el valle del Cauca (Colombia). Zoología-Taxonomía, Vol. 35, p. 199207. Recuperado de http://www.icn.unal.edu.co/

Niemczyk, E., y Kaźmierski, A. (2002). Checklist of species, occurrence and feeding of the members of families Stigmaeidae and Tydeidae in the apple orchards. Pp. 265-274, In: Postepy polskiej akarologii. (Ignatowicz, S., Ed.). SGGW, Warsaw, 274 pp. (In Polish). Recuperado de http://www.bioone.org/action/showPopup?citid=citart1\&id=bibr16\&doi=10.3161\%2F 000345409X432619 
Ochoa, R., Aguilar, H. y Vargas, C. (1991). Ácaros fitófagos de América Central: Guía ilustrada. CATIE. Turrialba, Costa Rica. p. 225

Pato, F. A., Condés, R. L. y Vicente, C. F. E. (2007). Introducción al cultivo del anthurium para flor cortada en la región de Murcia; p. 44

Rodríguez, R. M. D. (2007). Manejo integrado de plagas en cultivo de plantas en maceta. Horticultura Internacional, p. 64-66. Recuperado de http://www.horticom.com/revistasonline/horticultura/rhi59/64_67.pdf

San Juan, H. R. (2010). Dinámica socioeconómica en el agroecosistema ornamental con anturio (Anthurium andreanum L.) de la región Córdoba-Fortín de las Flores, Veracruz. Colegio de Postgraduados, CAMPUS Veracruz. Colegio de (Maestría en Ciencias). Tepetates, Manlio F. Altamirano, Veracruz. 